\title{
Effects of pupil filter patterns in line- scan focal modulation microscopy
}

\author{
Shuhao Shen \\ Shilpa Pant \\ Rui Chen \\ Nanguang Chen
}




\title{
Effects of pupil filter patterns in line-scan focal modulation microscopy
}

\author{
Shuhao Shen, ${ }^{\mathrm{a}}$ Shilpa Pant, ${ }^{\mathrm{b}}$ Rui Chen, ${ }^{\mathrm{a}}$ and Nanguang Chen ${ }^{\mathrm{a}, \star}$ \\ anational University of Singapore, Optical Bioimaging Laboratory, Department of Biomedical Engineering, Singapore \\ ${ }^{b}$ Nanyang Technological University, School of Electrical and Electronics Engineering, Centre for Optical Fibre Technology, Singapore
}

\begin{abstract}
Line-scan focal modulation microscopy (LSFMM) is an emerging imaging technique that affords high imaging speed and good optical sectioning at the same time. We present a systematic investigation into optimal design of the pupil filter for LSFMM in an attempt to achieve the best performance in terms of spatial resolutions, optical sectioning, and modulation depth. Scalar diffraction theory was used to compute light propagation and distribution in the system and theoretical predictions on system performance, which were then compared with experimental results. @ 2018 Society of Photo-Optical Instrumentation Engineers (SPIE) [DOI: 10.1117/1.JBO.23 3.036008]
\end{abstract}

Keywords: fluorescence microscopy; line-scan; pupil filter; modulation depth; scalar diffraction theory; microspheres. Paper 170585LRRR received Sep. 7, 2017; accepted for publication Feb. 27, 2018; published online Mar. 16, 2018.

Fluorescence microscopy is an indispensable tool for modern cell and tissue imaging. ${ }^{1}$ Most fluorescence microscopes can provide subcellular spatial resolutions for imaging living cells or tissues, ${ }^{2}$ which promotes the study of a wide range of biological processes inside the cells. The last few decades have witnessed the development of new fluorescence microscopy methods, which are based on complicated manipulation of light for biologic imaging. These advanced techniques include confocal microscopy and two-photon microscopy, which have been commercialized and are widely used in biological laboratories. Although they have high spatial resolution and molecular specificity, fluorescence microscopes are generally limited by their depth of penetration. Confocal microscopy achieves optical sectioning capability using a confocal pinhole; however, the effectiveness of the pinhole in eliminating out-of-focus light is compromised by scattering when imaging thick tissues. ${ }^{3,4}$ Multiphoton fluorescence microscopy can afford a higher imaging depth due to selective nonlinear excitation. Recently, deep imaging using three-photon microscopy has been extensively studied. A penetration depth beyond $1 \mathrm{~mm}$ in brain tissue has been reported. ${ }^{5}$ Some limitations of multiphoton microscopy are that the resolution is always slightly lower than singlephoton microscopy and the high cost of the short-pulse laser. ${ }^{6}$ In addition, nonlinear photo-damage and the limited availability of fluorescence probes are among other concerns. ${ }^{7}$

Focal modulation microscopy (FMM) is a recently developed optical microscopy technique ${ }^{8}$ that has huge potential in deep tissue imaging. It can provide submicron spatial resolution at large penetration depths in biological tissues by improved background rejection. ${ }^{9}$ To the best of our knowledge, FMM is the only one-photon microscopic technique that is able to achieve deep penetration in biological tissues for $>500$ microns. However, previously reported point-scanning FMM is limited by a relatively slow imaging speed. The first-generation FMM system, which used a mirror-based spatiotemporal phase modulator (STPM), could only achieve a minimum of $0.2-\mathrm{ms}$ pixel dwell time. Even after the development of fast STPMs, the imaging speed was still limited by the time consuming, point-to-point scanning process. ${ }^{9,10}$ The typical speed of 0.48 $\mathrm{fps}$ for an image size of $512 \times 512$ pixels is far from the requirement for visualizing fast biological processes that occur in the millisecond range. As a solution for in vivo microscopic imaging, we recently proposed a line-scanning version of the FMM system. ${ }^{11,12}$ Since one dimension of scanning is eliminated, an improvement in the temporal resolution can be achieved. LSFMM can readily achieve an imaging speed of $100 \mathrm{fps}(10 \mathrm{~ms}$ per image) for an image size of $500 \times 500$ pixels. $^{13}$

Extensive theoretical studies on a point-scan FMM system have been reported. ${ }^{14-16} \mathrm{~A}$ similar analysis regarding pupil patterns in a point-scanning FMM system revealed that special pupil filter designs determined the performance of FMM. ${ }^{17}$ However, theoretical studies on a pupil filter design in a linescan FMM system have not yet been explored. This letter focuses on the effects of pupil filter pattern applied to linescan FMM. Both theoretical studies and experimental verifications are carried out. System characterizations in terms of resolution, integrated intensity, and background rejection capability are presented and discussed. Finally, we use different pupil filter patterns to perform scattering beads image to evaluate the image quality of our LSFMM system.

Figure 1 shows the layout of our LSFMM system. The details of the experimental setup can be referred to in the previous publication. ${ }^{11}$ Particularly, the spatial polarizer (SP) here was a customer designed polarizer, where different zones passed one of the two different polarization modes (transmissive axis vertically or horizontally). This is where we generate different pupil filter patterns in practice. As the excitation beam is condensed one dimensionally in LSFMM, stripe-shaped pupil patterns become the only choice to meet the symmetry requirements.

In the theoretical study, we used the scalar Fresnel diffraction theory to analyze the light distribution in the LSFMM system. We first set up the parameters for the theoretical study. The 


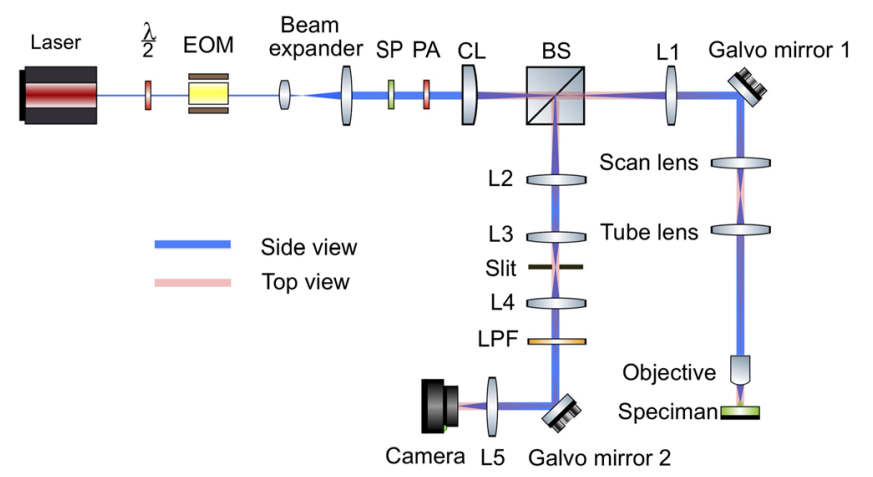

Fig. 1 Schematic diagram showing the optical setup for LSFMM: EOM, electro-optic modulator; SP, spatial polarizer; PA, polarization analyzer; $\mathrm{CL}$, cylindrical lens; $\mathrm{BS}$, beam splitter; LPF, long-pass filter. The focal length of $C L$ was $50 \mathrm{~mm}$. Focal lengths of lenses L1, L2, L3, L4, and L5 were 40, 40, 75, 75, and $35 \mathrm{~mm}$, respectively. The focal lengths of the scan lens and tube lens were 40 and $200 \mathrm{~mm}$, respectively.

wavelength of the light was set to $473 \mathrm{~nm}$ and the numerical aperture (NA) of the objective lens that we tried was 0.45 $(20 \times)$. In the image space, the effective slit width was set to $0.6 \mu \mathrm{m}$. For the illumination light path, the amplitude of the electric field in the focal region of a cylindrical lens illuminated by a plane wave was given by ${ }^{18}$

$h_{\mathrm{ill}}\left(v_{x}, u\right)=\int_{\text {pupil }} P(\xi) \exp \left(-\frac{i u \xi^{2}}{2}\right) \exp \left(-i v_{x} \xi\right) d \xi$

where $P(\xi)$ is the pupil function and the coordinate $\xi$ is the transverse displacement in the pupil plane, normalized by the pupil size. As the excitation beam was divided into a phase modulated half-beam and a nonmodulated half-beam, the pupil function $P(\xi)$ can be described as

$P(\xi)= \begin{cases}1, & \xi \in \text { non-modulated area } \\ \exp \left(i \frac{\pi+\pi \sin 2 \pi f t}{2}\right), & \xi \in \text { modulated area }\end{cases}$

Here, the fraction of the light field in the modulated area is sinusoidally modulated in time with frequency $f$ in contrast to the unmodulated fraction. The optical coordinates $\left(v_{x}, v_{y}, u\right)$ are defined by $v_{x}=2 \pi x \sin \alpha / \lambda, \quad v_{y}=2 \pi y \sin \alpha / \lambda, \quad u=$ $8 \pi z \sin ^{2}(\alpha / 2) / \lambda$, where $\lambda$ is the excitation wavelength, $\alpha$ is the semiangular aperture of the lens, and $x$ and $z$ are the distances measured from the focal point. Since the light intensity distribution pattern in the focus of a cylindrical lens is a uniform line, we extend the light field on the $x-z$ plane along the $y$-direction to obtain a uniform linear distribution in three-dimensional (3-D) space $h_{\text {ill }}\left(v_{x}, v_{y}, u\right)$.

For the detection path, we used circular apertures to detect the emission fluorescence light. For an aberration-free lens of a clear circular aperture, the electric field in the focal plane was given by ${ }^{19}$

$h_{\mathrm{det}}(v, u)=2 \int_{0}^{1} J_{0}(v \rho) \exp \left(-\frac{i u \rho^{2}}{2}\right) \rho d \rho$,

where $J_{0}$ denotes the zeroth-order Bessel function and $v=\sqrt{v_{x}^{2}+v_{y}^{2}}$ is the radial normalized optical coordinate.
The symbol $\rho$ is the radial displacement normalized by the pupil radius.

Using the scalar diffraction theory, we studied the 3-D intensity image of a point object to analyze the point spread function (PSF) of the LSFMM system. Neglecting the Stokes shift, the corresponding 3-D time-varying image of a point object with a finite size slit can be obtained from

$$
\begin{aligned}
I_{\mathrm{LSFMM}}\left(v_{x}, v_{y}, u\right)= & \left|h_{\mathrm{ill}}\left(v_{x}, v_{y}, u\right)\right|^{2} \times\left[\left|h_{\mathrm{det}}\left(v_{x}, v_{y}, u\right)\right|^{2}\right. \\
& \left.\otimes D\left(v_{x}\right)\right],
\end{aligned}
$$

where $\otimes$ denotes the one-dimensional convolution and $D\left(v_{x}\right)$ is the intensity sensitivity of the finite-sized slit. For a square pixellike detector in the sCMOS sensor array, the slit width was always set equal to the pixel size. The intensity sensitivity can be written as

$D\left(v_{x}\right)= \begin{cases}1, & \left|v_{x}\right| \leq v_{d} \\ 0, & \left|v_{x}\right|>v_{d}\end{cases}$

where $v_{d}$ is the normalized half width of the slit width, which is defined by $v_{d}=2 \pi r_{d} \sin \alpha / \lambda$, where $r_{d}$ is half the length of the slit width divided by the magnification of the overall optical detection system.

Modulation depth is an important parameter in FMM that indicates the efficiency of the FMM signal generation with respect to the total excitation loading on the sample. It is the ratio of the intensity of the modulated fluorescence signal (AC component) to the average intensity (DC component) collected by the detector, which is expressed as ${ }^{17}$

$M=\frac{\frac{1}{2}\left(I_{\max }-I_{\min }\right)}{\frac{1}{2}\left(I_{\max }+I_{\min }\right)}=\frac{\left(I_{\max }-I_{\min }\right)}{\left(I_{\max }+I_{\min }\right)}$.

When the phase over the apertures is uniform, the wellfocused excitation beam leads to the maximum detected signal $I_{\max }$. In contrast, when the relative phase delay of the modulated and nonmodulated beams is equal to $\pi$, a strong destructive interference will occur at the focus, which results in the minimum detected intensity $I_{\min } . I_{\max }$ and $I_{\min }$ can also be described using the equation

$\begin{aligned} I_{\max } & =\left.\iiint I_{\mathrm{LS}-\mathrm{FMM}}\left(v_{x}, v_{y}, u\right)\right|_{\varphi(t)=0} d v_{x} d v_{y} d u, \\ I_{\min } & =\left.\iiint I_{\mathrm{LS}-\mathrm{FMM}}\left(v_{x}, v_{y}, u\right)\right|_{\varphi(t)=\pi} d v_{x} d v_{y} d u,\end{aligned}$

where $\varphi(t)$ is the relative time-dependent phase delay between the modulated and nonmodulated regions.

To enhance the modulation depth, we need to search for the pupil filters that minimize $I_{\min }$. For an $n$-zone pupil filter, there are $n-1$ parameters $m_{1}, m_{2}, \ldots, m_{n-1}$, (normalized to the overall dimension of the pupil) that define the boundaries between modulated and nonmodulated zones. Our optimization process is confined to pupil filters with a maximal number of nine zones, which are practical in terms of the cost and accuracy to implement them. For a given number of zones, there exists an optimal set of boundary parameters for the modulation depth. Exhaustive searching for the optimal solution, nonetheless, is computationally economic only for a very small number of 
zones. Therefore, we resort to two intuitive approaches. The first is the maximally flat cater (MFC) method, which was previously developed for point-scanning FMM. ${ }^{20}$ The second is simply the equal-area (EA) patterns, which guarantee that the total areas of modulated and nonmodulated zones are identical and all off-center zones are equal in size. According to MFC, for a binary phase pupil filter with $n(n \geq 2)$ zones and $n-1$ boundaries at $m_{1}, m_{2}, \ldots, m_{n-1}$, the amplitude in the focal region can be expanded into a Taylor series with coefficients given by the moment of the aperture, where the $p$ 'th moment is defined as ${ }^{21}$

$$
\begin{aligned}
q_{p}= & \int_{-1}^{1} P(m) m^{p} d m \\
= & \frac{1}{p+1}\left[(-1)^{p+1}+2 m_{1}^{p+1}-2 m_{2}^{p+1}+\ldots\right. \\
& \left.+2(-1)^{n} m_{n-1}^{p+1}+(-1)^{n}\right] .
\end{aligned}
$$

The excitation intensity along the lateral and axial axes can be, respectively, expressed as

$$
\begin{aligned}
I\left(v_{x}\right) & =\left|h_{\mathrm{ill}}\left(v_{x}, 0\right)\right|^{2}=\left|\int_{-1}^{1} P(m) \exp \left(-i v_{x} m\right) d m\right|^{2} \\
& =\left|\sum_{p=0}^{\infty} \frac{(-i)^{p}}{p !} q_{p} v_{x}^{p}\right|^{2}
\end{aligned}
$$

and

$$
\begin{aligned}
I(u) & =\left|h_{\mathrm{ill}}(0, u)\right|^{2}=\left|\int_{-1}^{1} P(m) \exp \left(-\frac{i u m^{2}}{2}\right) d m\right|^{2} \\
& =\left|\sum_{p=0}^{\infty} \frac{(-i / 2)^{p}}{p !} q_{2 p} u^{p}\right|^{2} .
\end{aligned}
$$

To determine the $n-1$ unknowns (i.e., $m_{1}, m_{2}, \ldots, m_{n-1}$ ), we equate to zero the first $n-1$ moments of the aperture

$q_{p}=0, \quad p=0,1,2,3, \ldots, n-2$.

The MFC pupil filters can be readily found by solving these $n-1$ equations. In contrast, the EA pupil filter design is rather straightforward. EA patterns of two to nine zones are shown in Fig. 2. For EA with an even number of zones, all zones are the
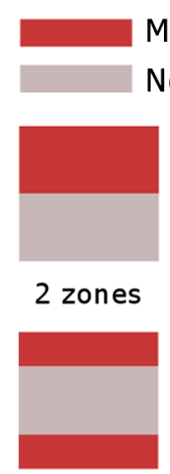

3 zones
Modulated

\section{Non modulated}
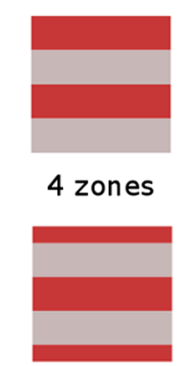

5 zones

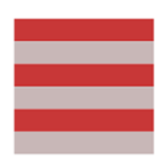

6 zones

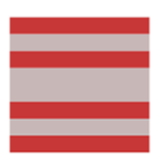

7 zones

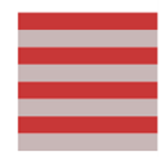

8 zones

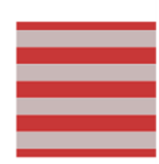

9 zones
Fig. 2 EA patterns (two to nine zones) of the LSFMM system. same in size. For EA with an odd number of zones, the central zone is twice as big as other zones.

Figure 3(a) compares the simulated modulation depth of LSFMM with EA and MFC pupil filters. Except for the identical case of two- and three-zone filters, EA always provides higher modulation depth than MFC for the same number of subapertures. To further demonstrate the performance of EA over MFC, we performed an exhaustive search for the maximal modulation depth for a four-zone pupil filter. As shown at the top of Fig. 3(b), the two variables $m_{1}$ and $m_{2}$ in the normalized pupil plane represent the possible boundary positions. The third boundary was fixed at the center due to symmetry considerations. The simulation results are displayed at the bottom of Fig. 3(b), which shows a maximum modulation depth at $m_{1}=-0.5, m_{2}=0.5$, which is exactly the EA pattern. This relationship is contrary to the previously reported point-scan FMM pupil designs. It has been found that, in the case of LSFMM, although the EA does not generate zero intensity around the center, the two main lobes of the point spread function (PSF) from EA are further separated from each other than MFC, as shown in Fig. 3(c). The deteriorated performance of MFC in the case of LSFMM can be explained using Eq. (10). For an $n$-zone MFC filter, the first few terms up to $v_{x}^{n-2}$ are set to zero. In contrast, in a point-scanning system the first few terms up to $v_{x}^{2 n-4}$ vanish due to the rotational symmetry of annular MFC filter. ${ }^{20}$ Therefore, MFC filters are expected to be less effective in pushing the intensity distribution away from the center in an LSFMM system. Given that EA outperforms MFC in LSFMM in terms of modulation depth, our further investigation is confined to EA pupil filters.

We first investigated the PSF in both line-scan confocal microscopy (LSCM) and LSFMM with EAs. Both lateral and axial resolutions are compared in Fig. 4(a) in terms of intensity profile along the $x, y$, and $z$-directions. With a two-zone pupil filter, LSFMM was able to achieve a significantly higher axial resolution than LSCM. For the lateral resolution, LSFMM and LSCM shared the same intensity distribution in the $y$-direction, but LSFMM provided higher resolution in the $x$-direction. To quantitatively characterize the effects of various pupil patterns with respect to resolution, we used the full-width at half maximum (FWHM) value to quantify the resolutions. Table 1 shows the FWHM values for several pupil patterns in LSFMM and also LSCM. The FWHMs in the lateral $y$-direction remains the same as LSCM at $0.711 \mu \mathrm{m}$ due to no modulation along the line focus, so we only compare the lateral resolutions in the $x$-direction and axial resolutions. We found that LSFMM with an odd number of zones has better axial resolutions than that with an even number of zones, whereas the lateral resolutions in the $x$-direction had no regular correlation with pupil patterns. Particularly, LSFMM with the pupil pattern of two zones had the highest lateral resolution (roughly $23 \%$ higher than LSCM), whereas LSFMM with a pupil pattern of three zones has the best performance in terms of axial resolution (around 16\% better than LSCM).

The resolution enhancement, however, is more or less a side benefit of LSFMM over LSCM. In the case of deep tissue imaging, the optical sectioning capability is a more important imaging performance indicator. In this letter, we use the integrated intensity to quantify the optical sectioning capability of LSFMM. The integrated intensity $I_{\text {int }}(u)$ can be expressed as $I_{\text {int }}(u)=\iint I\left(v_{x}, v_{y}, u\right) d v_{x} d v_{y}$ and $I\left(v_{x}, v_{y}, u\right)$ is the intensity point spread function (IPSF) for the microscopy system. 


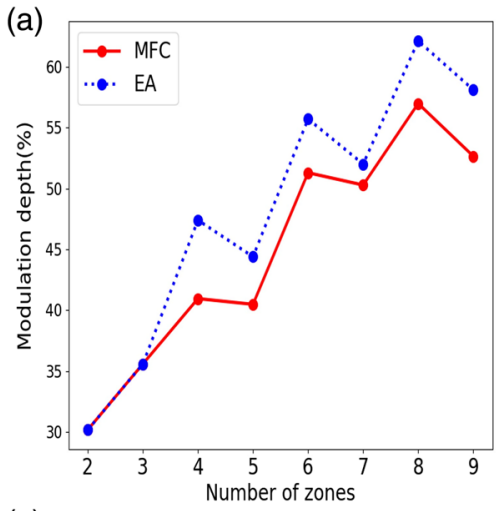

(b)
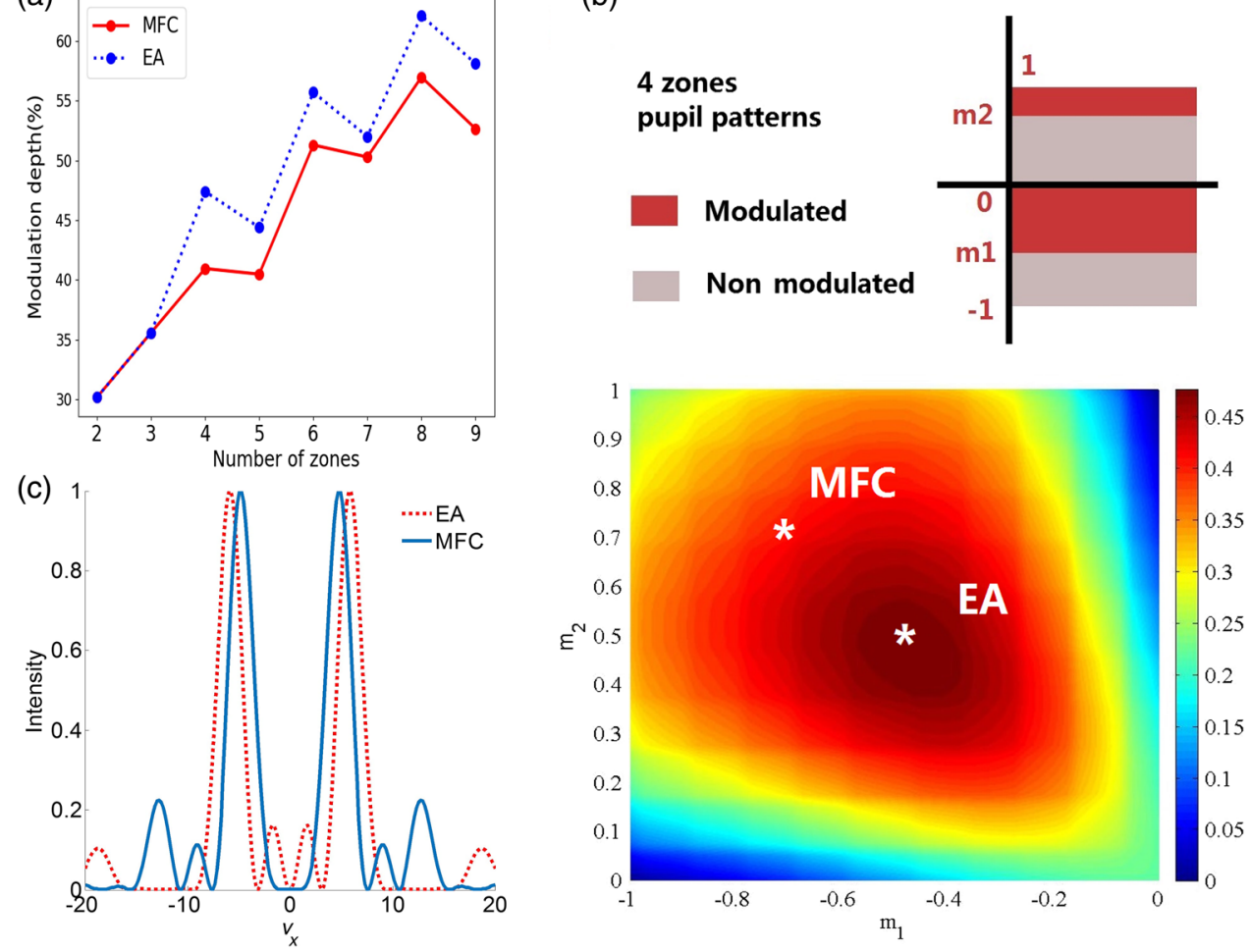

Fig. 3 (a) Modulation depth of LSFMM achieved with pupil filter patterns designed by MFC (blue dash) and EA (red solid), (b) modulation depth of LSFMM with pupil filter patterns containing four zones with two variable boundaries, and (c) intensity profile along the lateral direction of LSFMM with pupil filter patterns containing four zones.
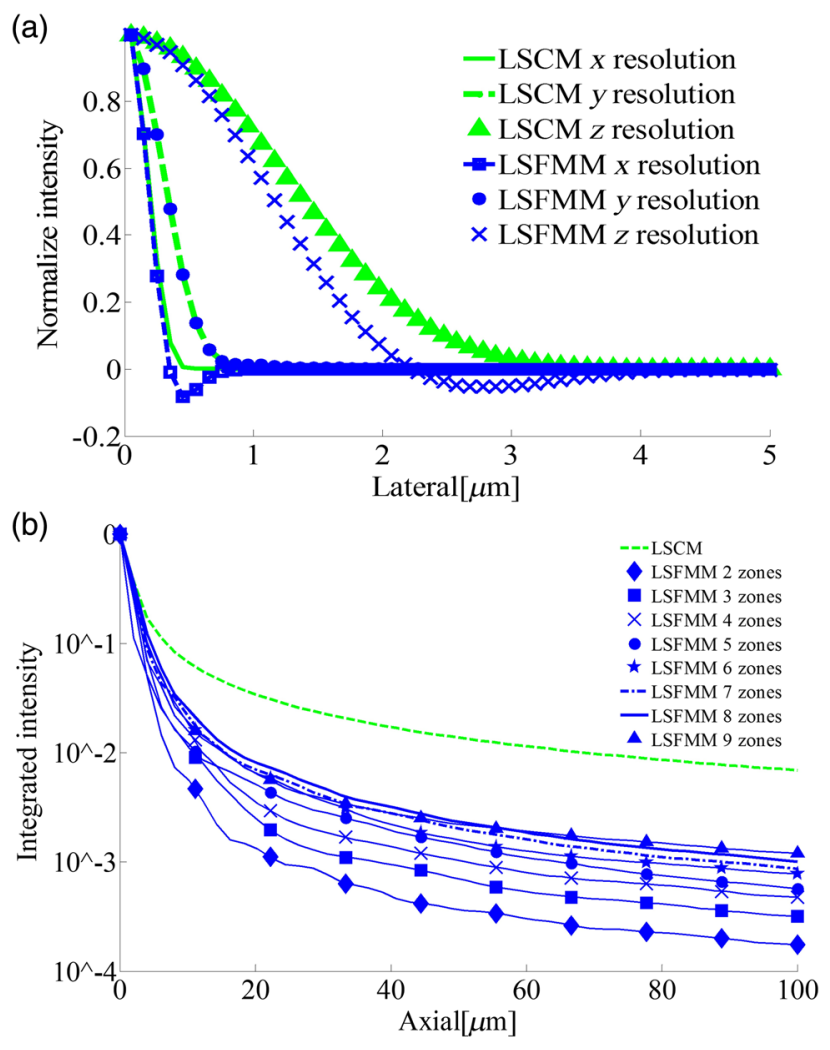

Fig. 4 (a) Intensity profile of the IPSF for LSFMM with a two-zone pupil filter and LSCM along lateral $(x, y)$ and axial $(z)$ directions and (b) decayed integrated intensity along the axial direction for LSCM and LSFMM with pupil filter patterns from two to nine zones.
Figure 4(b) is the $\log 10$ plot of the integrated intensity as a function of defocus distance in the axial direction. The pupil filter pattern with a narrow peak at the focus region is preferred because in this case the background light from outside the focal region will be suppressed. According to the plot, we can observe that the integrated intensity of LSFMM decays faster than LSCM as the axial depth increases. In addition, as the number of pupil filter zones decreases, the curve becomes sharper. In other words, pupil filter with fewer zones tend to provide better background rejection ability.

The simulated modulation depths were validated experimentally. Figure 5(a) shows the simulated and experimental modulation depths of all types of pupil filter patterns in an LSFMM system using a $20 \times$ objective lens $(\mathrm{NA}=0.45)$. With the number of zones increasing from 2 to 9 , the modulation depth increases from around $30 \%$ to $60 \%$. Pupil patterns with an even number of zones have higher modulation depth than those with an odd number of zones. A higher modulation depth is often desirable because it can afford a better signal to noise ratio.

To characterize the modulation depth in our experiments, Fluoresbrite $^{\circledR} \quad$ Carboxylate Yellow Green 1.0 Micron Microspheres from Polyscience (Catalogue No. 15702), which have excitation maxima at $441 \mathrm{~nm}$ and emission maxima at $486 \mathrm{~nm}$, were used. The beads were separated from each other by preparing a 1:1000 dilution of the beads in water. After that, the beads would be suspended in a solution containing 5\% agarose (Sigma, A9539). Agarose was used to immobilize the beads and also provided a transparent and nonscattering background during imaging. Figures 5(b) and 5(c) show the LSFMM raw images of the same $1-\mu \mathrm{m}$ fluorescent beads acquired with 
Table 1 Theoretical FWHM values in the lateral and axial directions for LSCM and LSFMM with different number of zones.

\begin{tabular}{|c|c|c|c|c|c|c|c|c|c|}
\hline FWHM & LSCM & LSFMM & LSFMM & LSFMM & LSFMM & LSFMM & LSFMM & LSFMM & LSFMM \\
\hline$(\mu \mathrm{m})$ & & 2 zones & 3 zones & 4 zones & 5 zones & 6 zones & 7 zones & 8 zones & 9 zones \\
\hline Lateral $x$ & 0.393 & 0.305 & 0.383 & 0.369 & 0.393 & 0.383 & 0.391 & 0.387 & 0.371 \\
\hline Axial & 2.791 & 2.779 & 2.329 & 2.787 & 2.727 & 2.789 & 2.665 & 2.791 & 2.619 \\
\hline
\end{tabular}

pupil filters of two and eight zones. From the raw images, we can observe the amplitude modulation of the focal signals along the $x$-direction. As shown in Fig. 5(d), the curves represent the intensity profiles in the two bead images along the red straight lines, which indicate the scanning direction. According to the experimental result, the modulation depth increases significantly from $29 \%$ to $63.1 \%$ when an eight-zone pupil filter replaces a two-zone pupil filter. After demodulation, the FMM signal enjoys a $6.75-\mathrm{dB}$ enhancement and so does the signal-to-noise ratio. However, the improvement over a six-zone pupil filter is marginal $(0.93 \mathrm{~dB})$.

To validate the optical sectioning capability of various pupil filters experimentally, we acquired images of 1-micron fluorescence beads inside in a turbid medium. Fluoresbrite ${ }^{\circledR}$ Carboxylate Yellow Green 1.0 Micron Microspheres were embedded in a scattering solution of 5\% ultrapure agarose (Invitrogen) and 4\% of lipofundin (diluted from 20\% Lipofundin MCT/LCT emulsion). As the scattering coefficient of lipofundin is close to most soft tissues in animals, ${ }^{22}$ this
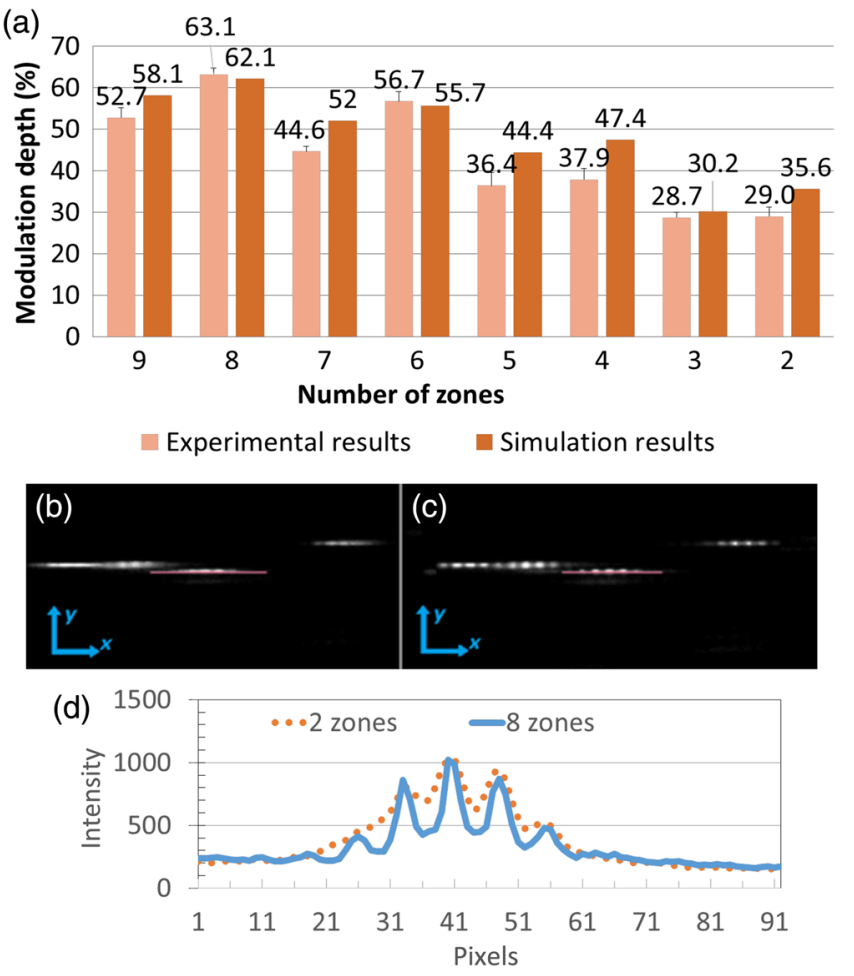

Fig. 5 (a) Comparison between experimental results and simulation results of modulation depth for $20 \times$ objective lens $(N A=0.45)$. $(b, c)$ LSFMM modulated images of the same $1-\mu \mathrm{m}$ fluorescent beads acquired with pupil filters of two and eight zones. (d) Intensity profile along one particular modulated bead indicated by red lines in (b) and (c). lipofundin bead sample is a good model for a highly scattering biological tissue. Shown in Figs. 6(a)-6(d) are images of the same $1-\mu \mathrm{m}$ bead acquired by LSCM and LSFMM with two-, four-, and eight-zones pupil filters, respectively. The image depth was $40 \mu \mathrm{m}$ away from the sample's surface. The intensity profiles along the bead indicated by yellow lines are plotted in Fig. 6(e). All the LSFMM images were reconstructed from raw images of normalized DC signals. It is evident that LSFMM images with various pupil filters are all much better than the LSCM image in terms of contrast and background level. However, comparing different LSFMM configurations is less straightforward. The peak intensity increases when the number of zones increases from two to four and then eight, which is consistent with our simulation results on modulation depth size (see Fig. 5). The background level, unfortunately, shows a similar trend, which agrees with the simulation results in Fig. 4(b). As a result, the signal-to-background ratios estimated from LSFMM images are 24.0, 25.5, and $22.5 \mathrm{~dB}$ for two-, four-, and eight-zone pupil filters, respectively.

To summarize, we have investigated the effect of the pupil filters in the LSFMM system. Both theoretical and experimental results suggest that the imaging performance of LSFMM depends on the STPM pupil design in a more complicated way than its point-scanning counterpart. With EA subapertures, a greater number of zones always lead to an improved signal level. However, the improvement becomes marginal when the number of zones exceeds six. In contrast, the signal-to-background ratio actually deteriorates when the number of zones
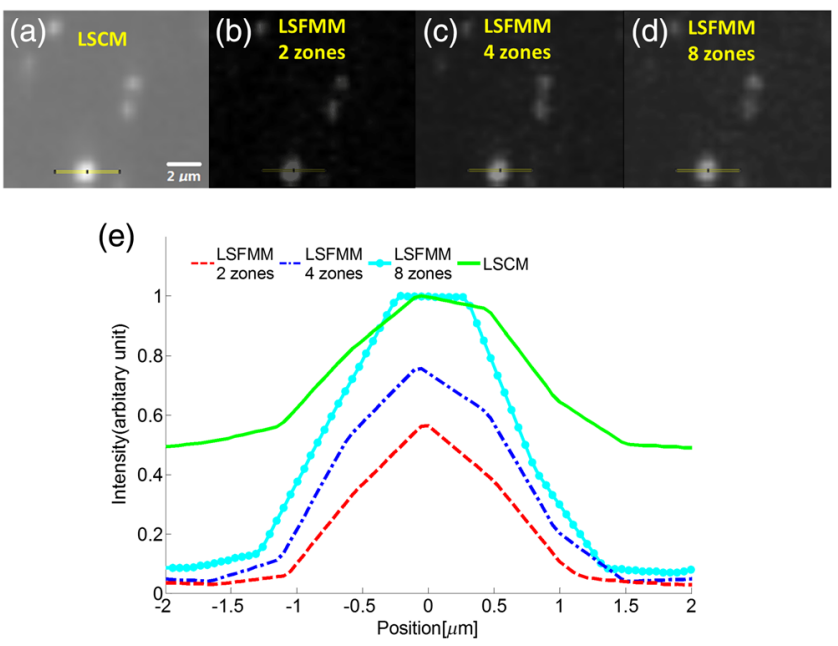

Fig. 6 Experimental images of $2-\mu \mathrm{m}$ scattering beads sample at image depth of $40 \mu \mathrm{m}$ acquired by (a) LSCM, (b) LSFMM with two zones pupil pattern, (c) LSFMM with four zones pupil pattern, (d) LSFMM with night zones pupil pattern, and (e) intensity profile along the bead indicated by the yellow lines in (a)-(d). 
reaches eight. In practice, the image quality afforded by four to eight zones is rather comparable when considering both signalto-noise ratio and signal-to-background ratio. As a side benefit, our simulation also suggests that LSFMM could provide higher resolution in both lateral and axial directions when a two- or three-zone pupil filter is used. This configuration can be applied to imaging of more transparent samples when the signal-tonoise ratio is less of a concern. We expect that this study can serve as guidance in pupil filters design for future LSFMM system development as well as those working on aperture coding in microscopy.

\section{Disclosures}

No conflicts of interest, financial or otherwise, are declared by the authors.

\section{Acknowledgments}

This work was supported in part by the National Medical Research Council (NMRC) Grants (NMRC/CBRG/0100/2015 and NMRC/CBRG/0036/2013) and internal funding from the National University of Singapore.

\section{References}

1. C. A. Combs and H. Shroff, "Fluorescence microscopy: a concise guide to current imaging methods," Curr. Protoc. Neurosci. 79, 2.1.1-2.1.25 (2010).

2. B. Alberts et al., "Looking at the structure of cells in the microscope," in Molecular Biology of the Cell, B. Alberts et al., Eds., pp. 547-570, Garland Science, New York (2002).

3. M. Gu, T. Tannous, and J. Sheppard, "Effect of an annular pupil on confocal imaging through highly scattering media," Opt. Lett. 21(5), 312-314 (1996).

4. J. Pawley and B. R. Masters, "Handbook of biological confocal microscopy," Opt. Eng. 35(9), 2765-2766 (1996).

5. N. G. Horton et al., "In vivo three-photon microscopy of subcortical structures within an intact mouse brain," Nat. Photonics 7(3), 205209 (2013).
6. P. T. So et al., "Two-photon excitation fluorescence microscopy," Аnnu. Rev. Biomed. Eng. 2(1), 399-429 (2000).

7. A. Hopt and E. Neher, "Highly nonlinear photodamage in two-photon fluorescence microscopy," Biophys. J. 80(4), 2029-2036 (2001).

8. N. Chen, C.-H. Wong, and C. J. Sheppard, "Focal modulation microscopy," Opt. Express 16(23), 18764-18769 (2008).

9. S. P. Chong et al., "High-speed focal modulation microscopy using acousto-optical modulators," Biomed. Opt. Express 1(3), 1026-1037 (2010).

10. N. Chen and G. Gao, "Multi-contrast focal modulation microscopy for in vivo imaging of thick biological tissues," Opt. Express 20(11), 12166-12170 (2012).

11. S. Pant et al., "Line-scan focal modulation microscopy," J. Biomed. Opt. 22(5), 050502 (2017).

12. N. Chen and S. Pant, "Line-scan focal modulation microscopy: a comparison study (Conference Presentation)," Proc. SPIE 9713, 971308 (2016).

13. S. Pant et al., "Augmented line-scan focal modulation microscopy for multi-dimensional imaging of zebrafish heart in vivo," Biomed. Opt. Express 8(12), 5698-5707 (2017).

14. W. Gong et al., "Focal modulation microscopy with annular apertures: a numerical study," J. Biophotonics 3(7), 476-484 (2010).

15. W. Gong et al., "Improved spatial resolution in fluorescence focal modulation microscopy," Opt. Lett. 34(22), 3508-3510 (2009).

16. B. Zhu et al., "Numerical studies of focal modulation microscopy in high-NA system," Opt. Express 24(17), 19138-19147 (2016).

17. G. Gao et al., "Considerations of aperture configuration in focal modulation microscopy from the standpoint of modulation depth," J. Opt. Soc. Am. A 28(4), 496-501 (2011).

18. C. J. Sheppard, "Cylindrical lenses-focusing and imaging: a review [Invited]," Appl. Opt. 52(4), 538-545 (2013).

19. M. Born and E. Wolf, Principles of Optics: Electromagnetic Theory of Propagation, Interference and Diffraction of Light, Elsevier, Amsterdam (1980).

20. Y. Duan et al., "Analytic method to optimize aperture design in focal modulation microscopy," Opt. Lett. 39(6), 1677-1680 (2014).

21. C. J. Sheppard, "Binary phase filters with a maximally-flat response," Opt. Lett. 36(8), 1386-1388 (2011).

22. S. L. Jacques, "Corrigendum: optical properties of biological tissues: a review,” Phys. Med. Biol. 58(14), 5007-5008 (2013).

Biographies for the authors are not available. 